\title{
Phenology and flowering overlap drive specialization in pollinator networks
}

1. Dept. of Environmental Science \& Policy, University of California-Davis, Davis, CA 95616

2. Dept. of Ecology \& Evolutionary Biology, University of Michigan, Ann Arbor, MI 48109

3. Laboratory of Zoology, University of Mons, Mons, 7000, Belgium

4. School for Environment and Sustainability, University of Michigan, Ann Arbor, MI 48109

14

* These authors contributed equally to this work and are listed alphabetically.

†Materials and Correspondence to:

17 Paul Glaum - prglaum@ucdavis.edu

Thomas J. Wood - thomasjames.wood@umons.ac.be

Classification: Article

Keywords: diet breadth, specialization, phenology, temporal resource density, pollination networks, 
53 have with their environment ${ }^{1-3}$. Consequently, understanding the drivers of this variation is key to

54 understanding ecological and evolutionary processes, and will facilitate the development of

55 predictive tools as ecological networks respond to environmental change ${ }^{4,5}$. Diet breadth in wild

56 bees has been an area of focus due to both their close mutualistic dependence on plants, and

57 because both groups are under threat from global biodiversity $\operatorname{loss}^{6}$. Though many of the principles

58 governing specialization for pollinators have been identified ${ }^{7,8}$, they remain largely unvalidated.

59 Using mechanistic models of adaptive foraging in pollinators ${ }^{9,10}$, we show that while temporal

60 resource overlap has little impact on specialization in pollinators with extended flight periods,

61 reduced overlap increases specialization as pollinator flight periods decrease. These results are

corroborated empirically using pollen load data taken from bees with shorter (genus Andrena) and longer (genus Lasioglossum) flight periods across environments with both high and low temporal resource overlap. This approach reveals how interacting phenologies structure plant-pollinator networks and drive pollinator diet breadth via the temporal overlap of floral resources. ecology and evolutionary biology. Diet breadth helps define organismal niches ${ }^{\mathbf{1}}$, mediates migration and dispersal potential ${ }^{2,11}$, shapes inter- and intraspecific competition ${ }^{\mathbf{3} 12}$, influences species persistence in the face of environmental disturbance ${ }^{4}$, and can affect rates of speciation and extinction ${ }^{5}$. Even the simple assembly of the collection of dietary ranges of organisms in a community defines one of the major concepts in ecology, the food web. Therefore, identifying the drivers influencing diet breadth and specialization provides fundamental steps towards understanding a multitude of essential biological questions.

Much of the progress in understanding specialization in consumers comes from studies of

75 herbivory ${ }^{1}$, particularly on insects ${ }^{\mathbf{1 3}}$. Using wild bees and plant-pollinator networks as our study system,

76 we extend the scope of those studies to include mutualistic interactions. A mutualism is a ubiquitous

77 ecological interaction in which participant species benefit each other ${ }^{14}$, and pollination therefore differs 
78 from other insect herbivory because of the reproductive benefit offered to both consumer and consumed

79 species. Bees depend on removing pollen from flowers to rear their offspring, providing a pollination

80 service at the same time ${ }^{15}$. Pollination is also of particular interest given its role in supporting terrestrial

81 biodiversity $^{6}$ and agricultural output ${ }^{16}$, a role threatened by widespread declines in both pollinators and

82 insect pollinated plants ${ }^{6,17}$.

83 Research on insect herbivores has largely focused on how adaptation to plant traits and defenses

84 drives consumer concentration on single plant families ${ }^{\mathbf{1 8 , 1 9}}$. However, other drivers of diet breadth have

85 been identified (ref 7,8, see Table S1). Here, we expand on the fundamental effect of resource density on

86 a consumer's diet breadth and specialization ${ }^{7}$, not in a spatial context, but temporally. This resource

87 density is important in its interaction with consumer phenology. For long-lived organisms (e.g., predatory

88 vertebrates), opportunities to access a single resource type principally depend on the resource's spatial

89 distribution. If a single resource type is physically dense, then a predator can specialize on it without

90 needing to turn to alternatives, whereas if it is patchily distributed then a strategy of specialization is less

91 optimal. However, for a comparatively short-lived organism like a bee whose adult flight period can be

92 measured in days, the distribution of a resource type in time is the principal determinant of accessibility.

93 A plant species that blooms for 30 days is de facto dense, continuously available resource for a bee

94 species whose flight period lasts for only 25 days, but sparse and patchily distributed for a species which

95 flies for 90 days. The temporal pattern of flowering can therefore be considered the functional equivalent

96 of resource density for short-lived organisms. These ideas have proven influential but require concrete

97 theoretical and empirical validation to solidify their conclusions.

Expanding upon this idea of temporal density, we hypothesize that the degree of temporal overlap

99 in the availability of different resources (flowering overlap among co-occurring plant species) in

100 combination with the fundamental life history phenologies of bees (adult flight period) determines

101 realized diet breadth. Applied to plant-pollinator systems, we hypothesize that reduced flowering overlap

102 will reduce the diet breadth of short-lived but not of long-lived pollinators (Fig. 1). Shorter-lived

103 pollinators will experience markedly different resource availability based on the degree of temporal 
104 overlap of co-flowering species. High flowering overlap will allow shorter-lived pollinators a greater

105 number of options while low overlap will restrict options. Longer-lived pollinators will be less constricted

106 by the temporal overlap of co-occurring resources, often having equal number of options regardless of the

107 degree of overlap (Fig. 1). Importantly, our hypothesis does not indicate that flight period will necessarily

108 correspond with being more or less specialized. Instead, it describes how temporal resource overlap will

109 affect diet breadth as a function of a pollinator's flight period.

110 We employed a multi-faceted approach to test our hypothesis. First, we leverage advances in

111 modeling adaptive foraging in ecological networks ${ }^{\mathbf{9} 10}$ to directly address the phenological mechanism

112 driving diet breadth across different pollinator flight periods. Second, we utilize our intercontinental

113 pollen load dataset of shorter lived Andrena bees and longer lived Lasioglossum bees from both a highly

114 seasonal and a less seasonal environment which function as low and high temporal resource overlap

115 treatments, respectively, to empirically validate our model outputs.

117 Results

118 Overview

119 Plant-pollinator dynamics are modeled using a dynamic consumer-resource approach that

120 incorporates adaptive foraging of pollinators to mechanistically model pollinators' consumption of floral

121 rewards and reproductive services to plant species ${ }^{9,10}$ (see Supplementary Methods Table S2). The

122 model's adaptive foraging mechanism serves as a useful tool to implement and measure changing

123 pollinator diet breadth (see Supplementary Methods). Phenology is integrated into the base model through

124 modified sinusoidal wave functions which produce unique phenologies for every plant $i\left(T_{p_{i}}\right)$ and animal

125 pollinator $j\left(T_{a_{j}}\right)$. These functions $T_{p_{i}}$ and $T_{a_{i}}$ regulate floral reward production, adaptive foraging rates,

126 pollinator visitation rates, and consequently, plant and pollinator reproduction across time (Tables S3-S4).

127 The malleability of our phenology functions facilitates direct control over the availability of specific

128 resources and the activity patterns of the animal pollinators over time in simulations (see Supplementary 
Methods, Fig S1-S5). The quantitative degree of overlap in co-occurring floral resources can be measured as the Total Resource Overlap (TRO) of the entire plant community, and the Averaged Resource Overlap (ARO) per plant species. Activity patterns of animal pollinators are set up to produce a range of different flight period lengths separated by differing lengths in between flight periods (Fig S6). Simulations use three fully connected bipartite network frameworks across 2072 combinations of plant and pollinator

134 phenologies to produce 62160 unique networks used to model over 22 million plant-pollinator

135 interactions (see Supplementary Methods).

Our pollen load data (see Supplementary Methods) comprises a large sample set of two bee genera, Andrena (Andrenidae) and Lasioglossum (Halictidae), collected both in the state of Michigan, USA (Nearctic) and the United Kingdom (Palearctic). Restricting our geographic scale to these two specific Holarctic locations aids in our ability to compare across both bee and plant communities due to their shared evolutionary history and overall similarity (see Supplementary Methods). The highly seasonal continental climate of Michigan ${ }^{20}$ produces lower degrees of overlap and highly seasonal

142 flowering communities, while the more mild oceanic climate of the British Isles ${ }^{\mathbf{2 0}}$ and its consistent 143 temperatures produces longer, overlapping flowering times among plant species ${ }^{21}$.

Diet breadth or the degree of specialization in pollinators is measured both in-model with output

145 on pollinator foraging effort and empirically with relative abundance of different pollen in bee pollen

146 loads. We used two metrics that were applicable to both theoretical and empirical results for better direct 147 comparisons, because simply counting unique taxonomic groups in the pollen data will not be applicable 148 to theoretical results. We developed the first metric and labeled it Deviation from Generalism (DFG) (see 149 Supplementary Methods, Table S5). Briefly, DFG is the normalized summation of all pairwise differences 150 of pollinator foraging effort (or relative pollen load) on potential floral resources (see Supplementary

151 Methods, Table S5). It ranges from 0 (perfect generalist) to 1 (perfect specialist). The second metric was 152 the Coefficient of Variation (CV) of a pollinator's foraging effort (or pollen load). Higher CV values 153 occur with outlying values indicating specialization. With model results, both DFG and CV were applied 
154 to final foraging levels at the end of simulations as well as the average foraging levels across the last 1000

155 time steps (see Supplementary Methods, Fig S7).

\section{Model Results}

Our simulation results (Figs. 2, 3) support our hypothesis (Fig. 1) demonstrating strong effects of temporal resource overlap on the diet breadth of short- but not long-lived pollinators. Fig. 2 shows the

159 results of one network as an illustration of how resource overlap explains large portions of the variation in

160 diet breadth of pollinators with shorter flight periods (Fig. 2a, c), but little for the diet breadth of

161 pollinators with longer flight periods (Fig. 2b, d). The transition from high explanatory power to low

162 explanatory power is apparent as pollinator flight period increases regardless if specialization was

163 measured at the end of simulations or averaged across the last 1000 model time steps (Fig 3). This result

164 is also consistent across varying proportional lengths of time between pollinator flight periods using

165 either the DFG or CV metric across all networks tested (Fig S8-Fig S10). As predicted, low levels of

166 resource overlap drive pollinators with short flight periods to specialize because potential resource

167 options are limited at any given moment in time. As temporal resource overlap increases, potential

168 options increase resulting in a more generalized diet breadth (Fig. 2a, c). Long-lived bees, on the other

169 hand, do not experience the same limitation of potential diet options due to temporal resource overlap,

170 because they are active most of the entire flowering season and can potentially access most or all of the

171 flowering plants. Results were consistent regardless of whether resource overlap was measured as total

172 resource overlap (TRO) or average resource overlap (ARO).

\section{Pollen data results}

Our empirical results support our hypothesis, showing more specialized diets of short-lived bees

175 in more seasonal environments (Michigan) with lower flowering overlap, than in less seasonal

176 environments (UK) with higher flowering overlap. Our pollen data from Michigan presents more single

177 family specialists than the less seasonal UK dataset (Fig S11). Dietary specialization between bee genera

178 and regions varied significantly at both the level of botanical family $\left(\chi^{2}=27.1, p<0.001\right.$, Fig $\left.4 a\right)$ and genus

$179\left(\chi^{2}=25.3, p<0.001\right.$, Fig $\left.4 b\right)$. In accordance with our hypothesis, there was an effect of region at the 
botanical family level, but only for the short-lived Andrena where species in Michigan were significantly more specialized than their British counterparts. In contrast, there were no differences for long-lived Lasioglossum. At the botanical genus level, the same trend was more strongly expressed, with Andrena in the UK showing a more generalized diet than their Michigan counterparts, but with no differences from Lasioglossum in either Michigan or the UK. Results were consistent when analyzed using the DFG metric (Fig S12).

\section{Discussion}

Our model results mechanistically demonstrate how differing phenologies interact with varying degrees of temporal resource overlap to alter pollinator diet breadth. These results are corroborated through our regional pollen load data which also presents the first empirical validation of benchmark theories on adaptive foraging by consumers in general $^{7}$ and pollinators in particular ${ }^{8}$. Furthermore, our results suggest broader implications regarding the drivers of bee diet breadth and diversification.

194 botanical family level ${ }^{13}$. These patterns have been linked to phytochemical diversity ${ }^{19,22,23}$ which

195 consequently drives an increase of both dietary specialization and species diversification in many

196 herbivorous insect groups across a latitudinal gradient towards the tropics, most clearly seen and well-

197 studied in Lepidoptera ${ }^{22,24}$. However, bees break sharply from this pattern, with a relatively low diversity

198 in the tropics when compared to Mediterranean and xeric environments ${ }^{\mathbf{8 , 2 5 , 2 6}}$. Tropical environments are

199 dominated by highly generalized, often social species that visit a huge variety of botanical families ${ }^{27,28}$.

200 Tropical areas have the highest levels of global phytochemical diversity, but their flowering patterns have

201 wider phenological variation than temperate areas at both the inter- and intraspecific levels. This results in

202 tropical floral communities that de facto show higher resource overlap in any particular year ${ }^{29,30}$ than

203 temperate communities. Our theoretical and empirical results suggest that bees' strong departure from the

204 typical global pattern in herbivory ${ }^{\mathbf{1 3}}$ may be due to a relatively stronger effect of phenology on their diet

205 breadth than the phytochemical restrictions suggested for other insect herbivores. In fact, tropical bees 
have not evolved anything approaching the same high degree of dietary specialization as seen in the tropical Lepidoptera despite existing in the same extremely phytochemically diverse landscapes. For further discussion of this point, see Supplementary Discussion. time dependent functional components into species behavior and traits show that even high dimensional network models can be further expanded in tractable ways to include the dynamics of organismal phenology. Additionally, our metrics for diet breadth complement each other (see Supplementary Methods) and reflect past methods (Fig S10). These metrics also integrated well with empirical pollen

214 load data sets that can be used to vet theoretical predictions. By expanding pollen datasets globally and

215 incorporating empirically vetted plant-pollinator phenology, future research can address how foraging

216 competition occurs across overlapping phenologies and what effects changing climatic conditions can

217 have on network stability.

218

Conflict of interest statement: We declare no conflict of interest.

Acknowledgements: Part of this project was supported by the National Science Foundation grant DEB1834497 awarded to F.S.V.

Author Contributions: F.S.V. conceived the project. T.J.W., P.G., F.S.V., and J.R.M. developed the conceptual basis for the project. T.J.W. identified suitable collection sites and bee genera for the empirical study design, collected bee and pollen data, and identified specimens. P.G. and F.S.V. developed dynamic model, simulation design, code, and diet breadth metrics. P.G. implemented simulations and analyses. T.J.W. and P.G. wrote the first draft, and all authors edited and revised the manuscript.

Supplementary Information: Supplementary Methods, Supplementary Discussion, Supplementary Figures and Tables.

Data Availability: Pollen load data is available as Supplementary Table S9.

Code Availability: Simulation code and simulation data are available at the repository: https://github.com/fsvaldovinos/Phenology. Phenology parameters used in the simulation portion of our study are available in Table S10.

\section{References}

1. Kartzinel, T.R. et al. DNA metabarcoding illuminates dietary niche partitioning by African large herbivores. PNAS 112(26), 8019-8024 (2015).

2. Bommarco, R., Biesmeijer, J.C., Meyer, B., Potts, S.G., Pöyry, J., Roberts, S.P., SteffanDewenter, I. \& Öckinger, E.Dispersal capacity and diet breadth modify the response of wild bees to habitat loss. Proc. Roy. Soc. B 277(1690), 2075-2082 (2010). 
3. Svanbäck, R. \& Bolnik, D.I. Intraspecific competition drives increased resource use diversity within a natural population. Proc. Roy. Soc. B 274(1161), 839-844 (2007).

4. Wood, T.J. et al. Narrow pollen diets are associated with declining Midwestern bumble bee species. Ecology 100(6) e02697 (2019).

5. Thompson, J. Rapid evolution as an ecological process. TREE 13(8), 329-332 (1998).

6. Biesmeijer, J.C., Roberts, S.P., Reemer, M., Ohlemüller, R., Edwards, M., Peeters, T., Schaffers, A.P., Potts, S.G., Kleukers, R., Thomas, C.D. \& Settele, J. Parallel declines in pollinators and insect-pollinated plants in Britain and the Netherlands. Science 313(5785), 351-354 (2006).

7. MacArthur, R.H. \& Pianka, E.R. On optimal use of a patchy environment. The American Naturalist 100(916), 603-609 (1966).

8. Waser, N.M., Chittka, L., Price, M.V., Williams, N.M. \& Ollerton, J. Generalization in pollination systems, and why it matters. Ecology 77(4), 1043-1060 (1996).

9. Valdovinos, F.S., Moisset de Espanés, P., Flores, J.D. \& Ramos-Jiliberto, R. Adaptive foraging allows the maintenance of biodiversity of pollination networks. Oikos 122(6), 907-917 (2013).

10. Valdovinos, F.S., Brosi, B.J., Briggs, H.M., Moisset de Espanés, P., Ramos-Jiliberto, R. \& Martinez, N.D. Niche partitioning due to adaptive foraging reverses effects of nestedness and connectance on pollination network stability. Ecology Letters 19, 1277-1286 (2016).

11. Boyle, W.A., Conway, C.J. \& Bronstein, J.L Why do some, but not all, tropical birds migrate? A comparative study of diet breadth and fruit preference. Evolutionary Ecology 25(1), 219-236 (2011).

12. deVries, M.S. The role of feeding morphology and competition in governing the diet breadth of sympatric stomatopod crustaceans. Biology Letters 13(4), 20170055 (2017).

13. Forister, M.L., Novotny, V., Panorska, A.K., Baje, L., Basset, Y., Butterill, P.T., Cizek, L., Coley, P.D., Dem, F., Diniz, I.R. and Drozd, P. The global distribution of diet breadth in insect herbivores PNAS 112(2), 442-447 (2015).

14. Bronstein, J.L. Our current understanding of mutualism. The Quarterly Review of Biology 69(1), 31-51 (1994).

15. Westerkamp, C. Pollen in bee-flower relations. Some considerations on melittophily. Bot. Acta. 109, 325-332 (1996).

16. Garibaldi, L.A., Steffan-Dewenter, I., Winfree, R., Aizen, M.A., Bommarco, R., Cunningham, S.A., Kremen, C., Carvalheiro, L.G., Harder, L.D., Afik, O. \& Bartomeus, I. Wild pollinators enhance fruit set of crops regardless of honey bee abundance. Science 339, 1608-1611 (2013).

17. Burkle, L. A., Marlin, J. C., \& Knight, T. M. Plant-pollinator interactions over 120 years: loss of species, co-occurrence, and function. Science 339 (6127), 1611-1615 (2013).

18. Ali, J.G. \& Agrawal, A.A. Specialist versus generalist insect herbivores and plant defense. Trends in Plant Science 17(5), 293-302 (2012).

19. Hunter, M. The Phytochemical Landscape: Linking Trophic Interactions and Nutrient Dynamics. Princeton University Press, 376 pp (2016).

20. Beck, H., Zimmermann, N., McVicar, T. et al. Present and future Köppen-Geiger climate classification maps at 1-km resolution. Sci Data 5, 180214 https://doi.org/10.1038/sdata.2018.214 (2018).

21. Baude, M., Kunin, W.E., Boatman, N.D., Conyers, S., Davies, N., Gillespie, M.A., Morton, R.D., Smart, S.M. \& Memmott, J. Historical nectar assessment reveals the fall and rise of floral resources in Britain. Nature 530, 85-88 (2016).

22. Becerra, J. On the factors that promote the diversity of herbivorous insects and plants in tropical forests. PNAS 112(19), 6098-6103 (2015).

23. Richards, L.A., Dyer, L.A., Forister, M.L., Smilanich, A.M., Dodson, C.D., Leonard, M.D. \& Jeffrey, C.S. Phytochemical diversity drives plant-insect community diversity. PNAS 112(35), 10973-10978 (2015). 
24. Dyer, L.A., Singer, M.S., Lill, J.T., Stireman, J.O., Gentry, G.L., Marquis, R.J., Ricklefs, R.E., Greeney, H.F., Wagner, D.L., Morais, H.C. \& Diniz, I.R. Host specificity of Lepidoptera in tropical and temperate forests. Nature 448(7154), 696-699 (2007).

25. Heithaus, E.R. Community structure of neotropical flower visiting bees and wasps: diversity and phenology. Ecology 60(1), 190-202 (1979).

26. Moldenke, A.R. The role of host-plant selection in bee speciation processes. Phytologia, 43, 433460 (1979).

27. Eltz, T., Brühl, C.A., van der Kaars, S., Chey, V.K. \& Linsenmair, K.E. Pollen foraging and resource partitioning of stingless bees in relation to flowering dynamics in a Southeast Asian tropical rainforest. Insectes sociaux 48, 273-279 (2001).

28. Galgani-Barraza, P., Moreno, J.E., Lobo, S., Tribaldos, W., Roubik, D.W. \& Wcislo, W.T. Flower use by late nineteenth-century orchid bees (Eufriesea surinamensis, Hymenoptera, Apidae) nesting in the Catedral Basílica Santa María la Antigua de Panamá. Journal of Hymenoptera Research 74, 65-81 (2019).

29. Newstrom, L.E., Frankie, G.W. \& Baker, H.G. A new classification for plant phenology based on flowering patterns in lowland tropical rain forest trees at La Selva, Costa Rica. Biotropica 26, 141-159 (1994).

30. Stevenson, P.R., Castellanos, M.C., Cortés, A.I. \& Link, A. Flowering patterns in a seasonal tropical lowland forest in western Amazonia. Biotropica 40, 559-567 (2008). 


\section{Figures \& Figure Legends}

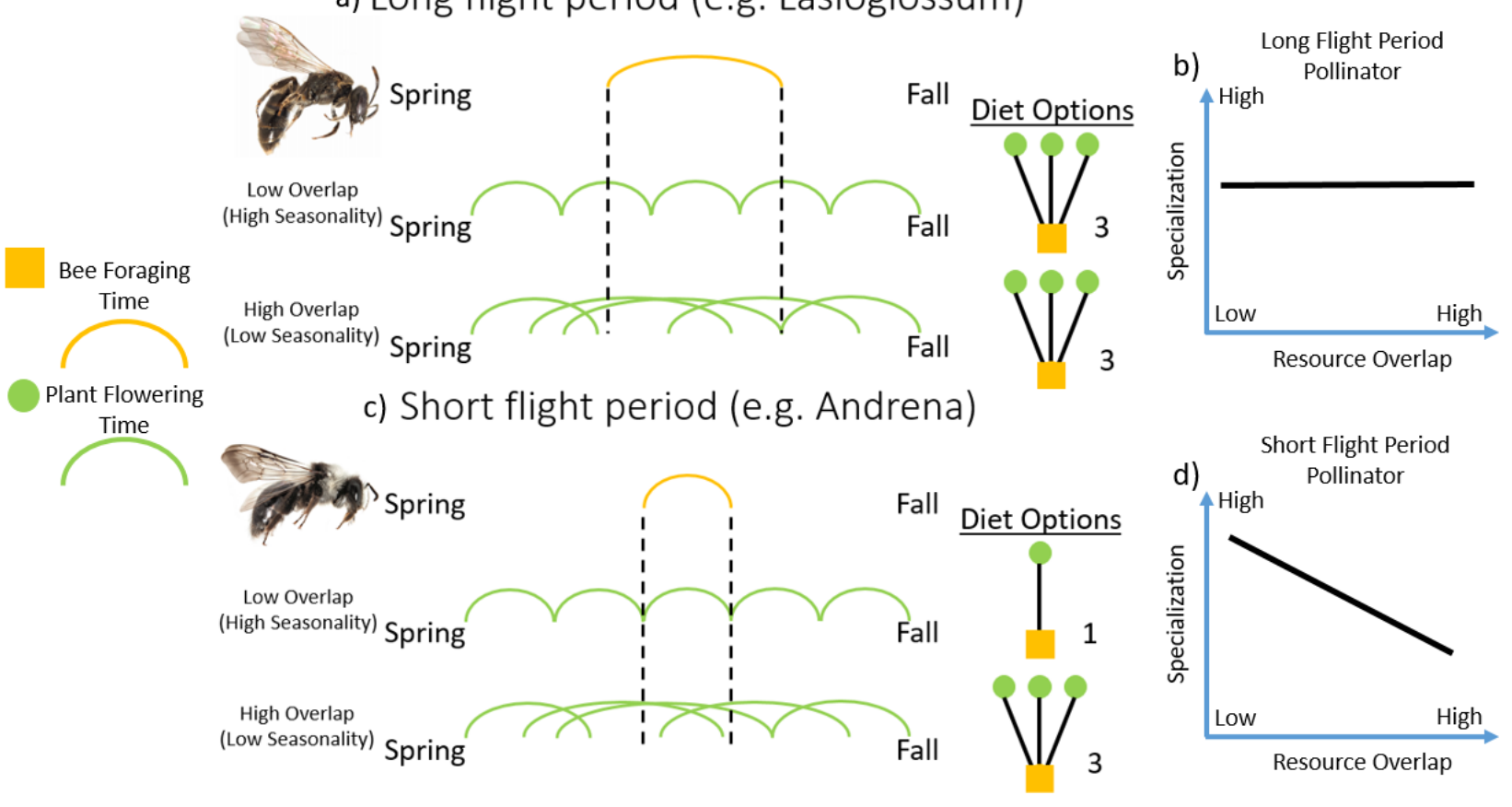

Figure 1: Graphical diagram of our hypothesis. Our hypothesis stems from the temporal overlap of flowering resources available to pollinators. Highly seasonal delimitations between different flowering populations create low levels of temporal overlap amongst different flowering resources. On the other hand, fewer seasonal delimitations between different flowering populations create higher levels of temporal overlap amongst different flowering resources. a) In long flight-period pollinators (e.g. Lasioglossum), differing levels of temporal resource overlap have limited to no effect on the degree of diet options available to the pollinator. $b$ ) This is predicted to lead to a non-significant relationship between overlap and specialization in long flight period pollinators. c) In short flight-period pollinators (e.g. Andrena), low and high temporal resource overlap can limit or expand pollinator diet options respectively. d) This is predicted to lead to a significant relationship between overlap and specialization in short flight-period pollinators. 

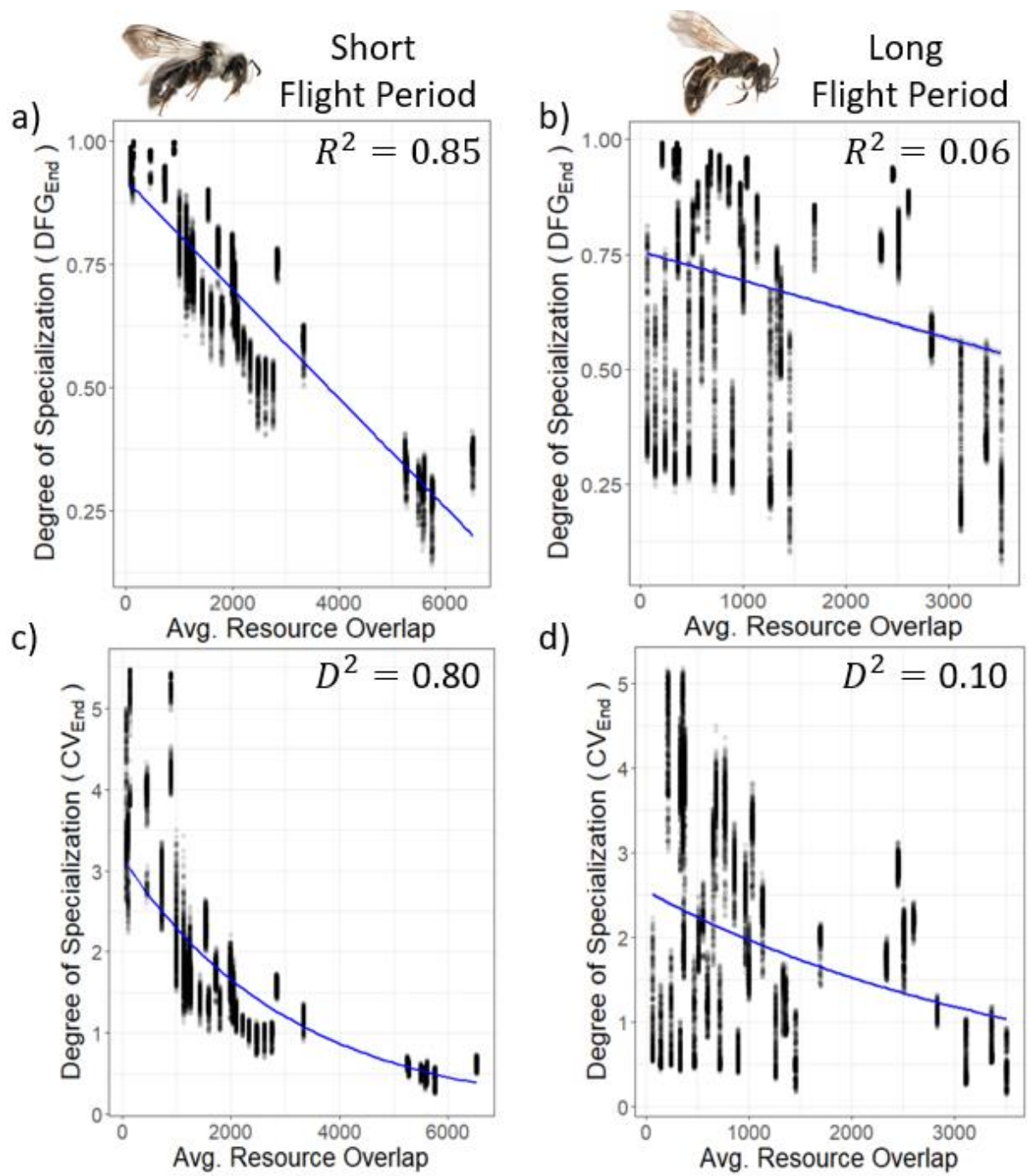

Figure 2: Temporal resource overlap drives specialization more strongly in short-lived than in longlived pollinators. Model output on pollinator specialization taken across average plant overlap (ARO) for short flight period (flight period $=20$ time steps) and long flight period pollinators (flight period $=300$ time steps). Each dot represents the diet breadth metric of a single pollinator population based on their foraging effort per plant species. Diet breadth was measured at the end of simulations using a) DFG in a short flight period bee, F-statistic: $1.05 \mathrm{e}+05$ on 1 and $18498 \mathrm{DF}$, p-value: $<2.2 \mathrm{e}-16, \mathbf{b}) \mathrm{DFG}$ in a long flight period bee, F-statistic: 1089 on 1 and 18498 DF, p-value: <2.2e-16, c) CV in a short flight period bee, Residual deviance: 671.94 on 18498 degrees of freedom, d) CV in a long flight period bee, Residual deviance: 8244.4 on 18498 degrees of freedom. Example data taken from a network framework with 30 plant and 50 pollinator species. Higher values of both DFG and CV metrics indicate higher levels of specialization (narrower diet breadth) while lower values indicate a more generalized feeding range

(wider diet breadth). 

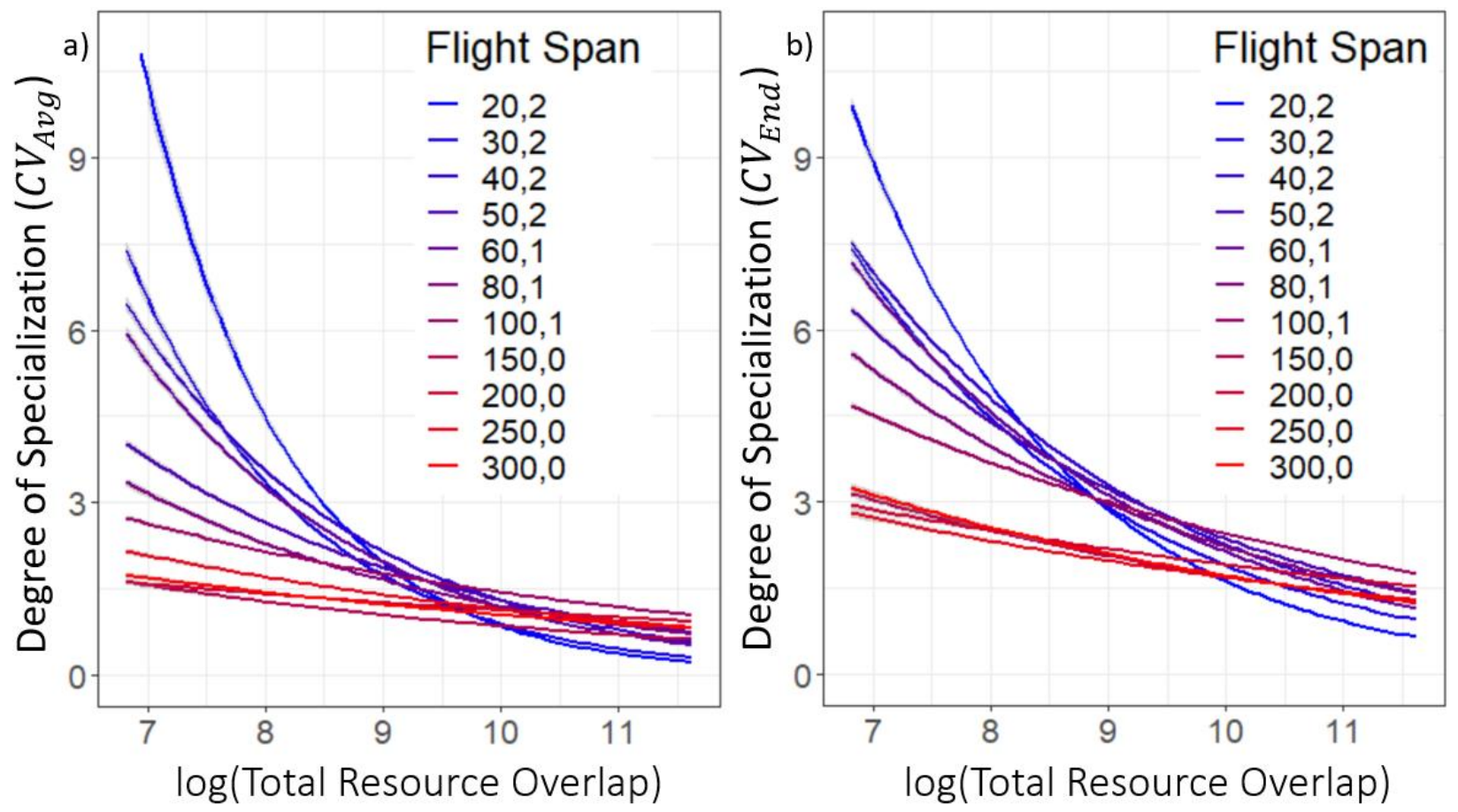

Figure 3: Interactive effect of temporal resource overlap and pollinator flight period on pollinator diet breadth. Representative sample of generalized linear models (Gamma distribution with log link functions) showing change in effect of Total Resource Overlap (TRO) on pollinator diet breadth in model simulations. Flight period labels indicate the length of the flight period and the number of phenology cycles between subsequent flight periods (see Supplementary Methods). Diet breadth shown as the degree of specialization measured by the $\mathrm{CV}$ of pollinator foraging effort per plant species taken a) at the end of simulations and b) averaged across the last 1000 time steps. Higher CV values correspond with a higher level of specialization (narrower diet breadth) while lower CV values indicate a more generalized feeding range (wider diet breadth). Example data taken from a network framework with 30 plant and 50 pollinator species. 
a)

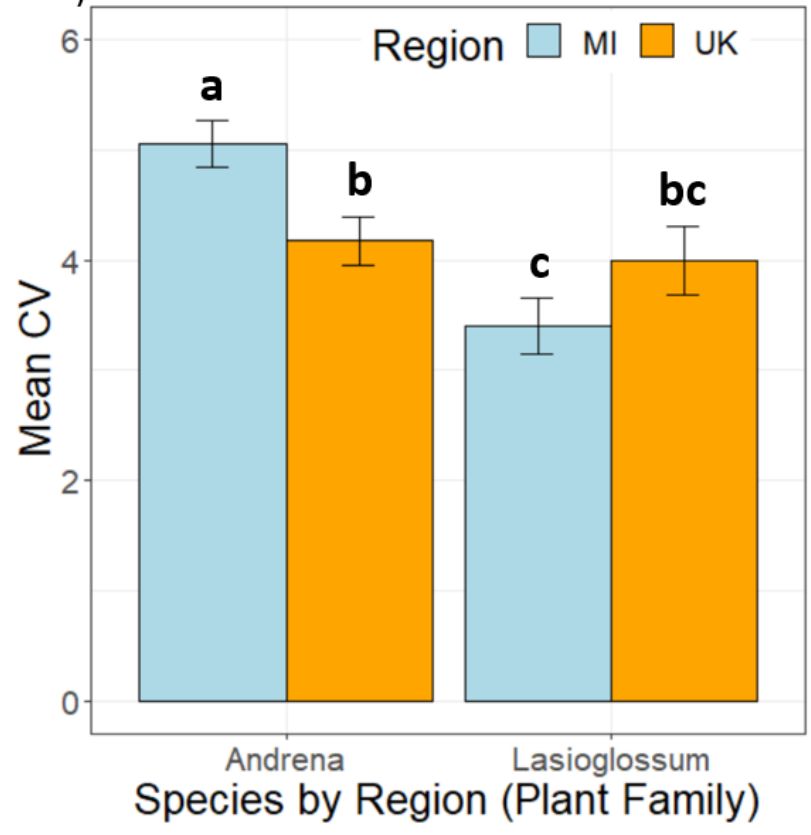

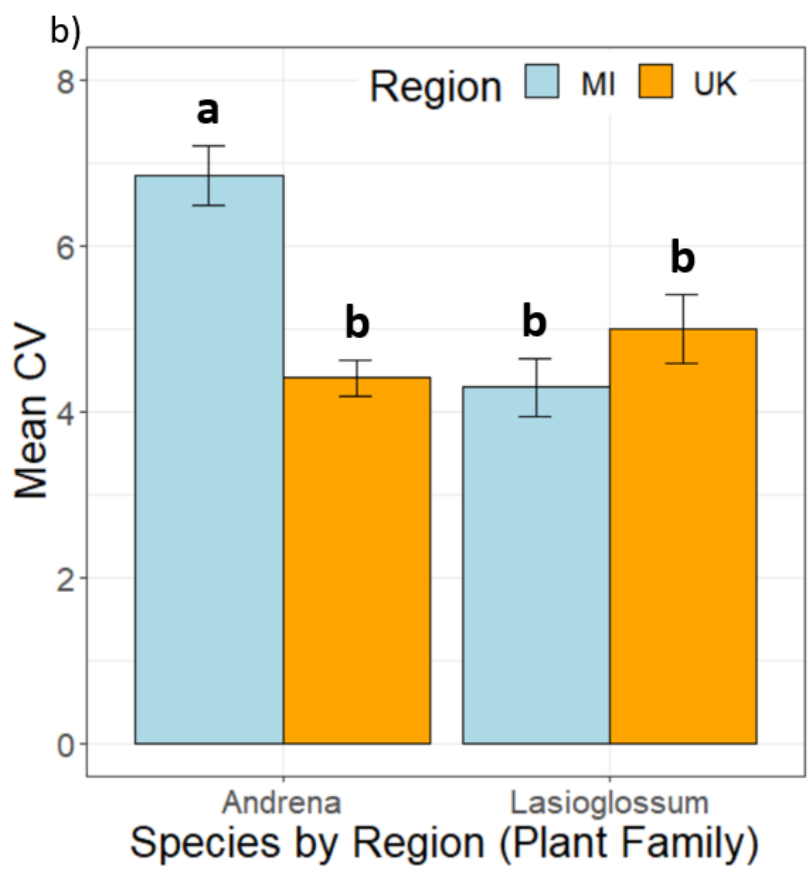

Figure 4: Empirical measures of dietary specialization. Short-lived Andrena bees show higher levels of specialization in highly seasonal (Michigan) compared to the weakly seasonal (UK), with no differences for long-lived Lasioglossum bees using the CV metric. Moreover, only in Michigan is there a difference in specialization between the two bee genera, showing a minimal impact of flight period in weakly seasonal environments. Effects are consistent at both the (a) botanical family and (b) botanical 